\title{
Neural Correlates of Impulse Control During Stop Signal Inhibition in Cocaine-Dependent Men
}

\author{
Chiang-shan Ray $\mathrm{Li}^{1}{ }^{1 *}$, Cong Huang ${ }^{1,2}$, Peisi Yan ${ }^{1,2}$, Zubin Bhagwagar', Verica Milivojevic' and Rajita Sinha' \\ 'Department of Psychiatry, Yale University, New Haven, CT, USA; ' ${ }^{2}$ Department of Statistics, Yale University, New Haven, CT, USA
}

\begin{abstract}
Altered impulse control is associated with substance use disorders, including cocaine dependence. We sought to identify the neural correlates of impulse control in abstinent male patients with cocaine dependence (PCD). Functional magnetic resonance imaging (fMRI) was conducted during a stop signal task that allowed trial-by-trial evaluation of response inhibition. Fifteen male PCD and I5 healthy control $(H C)$ subjects, matched in age and years of education, were compared. Stop signal reaction time (SSRT) was derived on the basis of a horse race model. By comparing PCD and HC co-varied for stop success rate, task-related frustration rating, and post-error slowing, we isolated the neural substrates of response inhibition, independent of attentional monitoring (of the stop signal) and post-response processes including affective responses and error monitoring. Using region of interest analysis, we found no differences between $\mathrm{HC}$ and PCD who were matched in stop signal performance in the pre-supplementary motor area (pre-SMA) previously shown to be associated with SSRT. However, compared with HC, PCD demonstrated less activation of the rostral anterior cingulate cortex (rACC), an area thought to be involved in the control of stop signal inhibition. The magnitude of $r A C C$ activation also correlated negatively with the total score and the impulse control subscore of the Difficulty in Emotion Regulation Scale in PCD. The current study thus identified the neural correlates of altered impulse control in PCD independent of other cognitive processes that may influence stop signal performance. Relative hypoactivation of the $\mathrm{AACC}$ during response inhibition may represent a useful neural marker of difficulties in impulse control in abstinent cocaine-dependent men who are at risk of relapse.

Neuropsychopharmacology (2008) 33, 1798-1806; doi: I0. 1038/sj.npp. I 301568; published online 26 September 2007
\end{abstract}

Keywords: prefrontal; frontal; executive; psychostimulant; cognitive control; inhibitory control

\section{INTRODUCTION}

Deficits in inhibitory control or impulsivity have been thought to directly or indirectly influence substance use behaviors (Ernst and Paulus, 2005). Substance dependence, characterized by impulsive drug seeking and consumption, is distinctly captured by a loss in impulse control (Everitt and Robbins, 2005; Goldstein and Volkow, 2002). Studies employing different personality scales to measure impulse control have demonstrated greater impulsivity in individuals with substance use disorders (SUD) including those with cocaine dependence (Dawe and Loxton, 2004). Moreover, impaired impulse control is associated with poor treatment outcome and relapse, adverse life events, and suicidal behaviors in patients with SUD (Dougherty et al, 2004; Hayaki et al, 2005; Moeller et al, 2001).

However, relatively little is known about the neural substrates underlying impaired impulse control in patients

* Correspondence: Dr C-SR Li, Connecticut Mental Health Center, SI03, Department of Psychiatry, Yale University School of Medicine, 34 Park Street, New Haven, CT 06519, USA, Tel: + I 203974 7354, Fax: + I 203974 7076, E-mail: chiang-shan.li@yale.edu

Received 16 May 2007; revised 8 August 2007; accepted 9 August 2007 with SUD. Behavioral tasks that examine impulse control oftentimes involve perception, attention, working memory, and post-response processing, which are mediated by distinct regions of the frontal cortex and can influence the measure of response inhibition. While previous research has shown altered activation in several regions of the frontal cortex in patients with SUD, whether this frontal dysfunction is specific to impulse control or related to other cognitive and affective processes is unknown (Hester and Garavan, 2004; Kaufman et al, 2003). For instance, a previous functional magnetic resonance imaging (fMRI) study employing a 'go/no-go' task reported attenuated response in the pre-supplementary motor area (pre-SMA) and insula both during successful 'no-go' trials and commission errors in cocaine-dependent patients, compared to cocaine-naive controls (Kaufman et al, 2003). However, since less attentional monitoring for the 'no-go' signal could lead directly to commission errors and commission errors evoke affective responses and cognitive compensatory processes, it was not clear whether the regional frontal hypoactivation truly reflected a deficit in impulse control. Furthermore, whether hypoactivation during impulse control tasks are in fact associated with subjective and functional reports of difficulties in impulse control has not been examined. 
We previously established an algorithm to address cognitive and affective processes associated with impulse control (Li et al, 2005c, 2006c). In this research we isolated the behavioral and neural measure of inhibitory control using a tracking stop signal task. To this end, a number of task-related factors such as attention and error monitoring that can influence stop signal performance were identified, in addition to the performance measure on inhibitory control-the stop signal reaction time (SSRT), which was obtained on the basis of the horse race model ( $\mathrm{Li}$ et al, 2006a; Logan, 1994). Using fMRI, we identified two specific regions, a prefrontal and an anterior cingulate locale, which appear to mediate response inhibition independent of these other task-related factors (Li et al, 2006a). The dorsal medial frontal cortex $(\mathrm{dmFC})$ and the rostral anterior cingulate cortex (rACC) showed greater activation in observers with short SSRT, compared to those with long SSRT. Furthermore, activation of the dmFC correlated with SSRT, consistent with its role in withholding the response, while activation of the rACC did not correlate with SSRT, consistent with its role in controlling response inhibition. These two brain regions thus each played a motor-level and control-level role in stop signal inhibition, independent of other cognitive and affective processes (Li et al, 2006a, b). In the current study, we examine the specific deficit in regional brain activation during response inhibition that may be associated with chronic cocaine abuse. On the basis of our previous work, the neural correlates of response inhibition were studied after accounting for the effects of attentional signal monitoring and post-response processing. We hypothesized that, compared to healthy individuals, patients with cocaine dependence (PCD) would demonstrate decreased rACC activation during response inhibition. Additionally, since rACC hypoactivation has been implicated in deficient executive control in patients with stimulant use, we expected that such rACC hypoactivation would be associated with subjective difficulties in impulse control in PCD (Aron and Paulus, 2007; Jovanovski et al, 2005; Sinha et al, 2005; Verdejo-García et al, 2006). Men and women demonstrate important differences in their drug using behaviors and in their clinical profiles of SUD (Brady and Randall, 1999; Sinha and Rounsaville, 2002). Furthermore, significant sex differences in brain responses have also been demonstrated in both healthy individuals and patients with SUDs (Adinoff et al, 2006; Goldstein et al, 2005; Kim et al, 2005; Li et al, 2005a, b, 2006b; Wager et al, 2003). In particular, women appear to involve multiple neural processes during stop signal inhibition, such that an increased number of subjects and perhaps an alternative experimental design would be required to examine altered frontal processes in cocaine dependent, compared to healthy control (HC), females ( $\mathrm{Li}$ et al, 2006b). We, therefore, tested the proposed hypotheses only in men in the current study.

\section{MATERIALS AND METHODS}

\section{Subjects, Informed Consent, and Assessment of Impulse Control}

Fifteen male abstinent PCD and age- and educationmatched HC men participated in the study (Table 1). PCD met criteria for current cocaine dependence, as diagnosed by the Structured Clinical Interview for DSM-IV (First et al, 1995). Recent cocaine use was confirmed by urine toxicology screens upon admission. They were drug-free for a minimum of 2 weeks while staying in an inpatient treatment unit prior to the current fMRI study. All subjects were physically healthy with no major medical illnesses or current use of prescription medications. None of them reported having a history of head injury or neurological illness. Other exclusion criteria included dependence on another psychoactive substance (except nicotine) or past history of any substance abuse/dependence (except nicotine) and current or past history of psychotic disorders. Individuals with current depressive or anxiety symptoms requiring treatment or currently being treated for these symptoms were excluded as well. The Human Investigation committee at Yale University School of Medicine approved all study procedures, and all subjects signed an informed consent prior to study participation.

Because PCD are known to have impaired impulse and affective control, we assessed their difficulties in emotion regulation and impulse control using the Difficulties in Emotion Regulation Scale (DERS) weekly during their inpatient stay (Gratz and Roemer, 2004). The DERS is a 36 -item self-report measure, with each item rated on a 5 -point analog scale: $1=$ almost never; $2=$ sometimes;

Table I Demographics of the Subjects

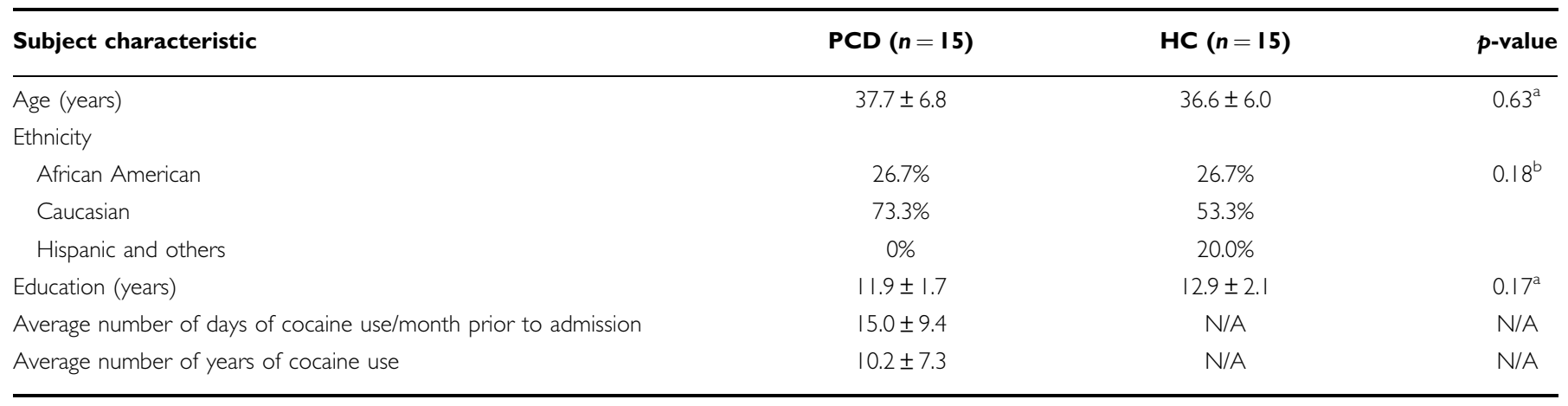

Abbreviations: $\mathrm{HC}=$ healthy control; N/A, not applicable; $\mathrm{PCD}=$ patients of cocaine dependence.

Note: values are mean \pm SD.

${ }^{a}$ Two-sample $t$-test.

${ }^{b} \chi^{2}$ test. 
$3=$ about half of the time; $4=$ most of the time; $5=$ almost always. It has been shown to have high internal consistency, test-retest reliability, and construct validity in the general population and in cocaine-dependent patients (Fox et al, 2007; Gratz and Roemer, 2004). Six subscales have been identified and the impulse control difficulty subscale contained 6 items: when I'm upset, I lose control over my behaviors; when I'm upset, I have difficulty controlling my behaviors; when I'm upset, I become out of control; when I'm upset, I feel out of control; I experience my emotion as overwhelming and out of control; when I'm upset, I feel like I can remain in control of my behaviors (reverse-scored). The total score of DERS ranges from 36 to 180 and the impulse control subscale score ranges from 6 to 30, with higher score indicating greater emotion dysregulation and difficulties in impulse control. Abstinent cocaine patients were found to have persistent problems in impulse control compared to healthy individuals (Fox et al, 2007).

\section{Behavioral Task}

We employed a simple reaction time (RT) task in this stop signal paradigm (Figure 1). There were two trial types: 'go' and 'stop,' randomly intermixed. A small dot appeared on the screen to engage attention and eye fixation at the beginning of a go trial. After a randomized time interval (fore-period) anywhere between 1 and $5 \mathrm{~s}$, the dot turned into a circle, which subtended approximately $2^{\circ}$ of visual angle. The circle served as an imperative stimulus and the subjects were instructed to quickly press a button at the 'go' signal but not before. The circle vanished at button press or after $1 \mathrm{~s}$ had elapsed, whichever came first, and the trial was terminated. A premature button press prior to the appearance of the circle also terminated the trial. Three quarters of all trials were 'go' trials. In a stop trial, an additional ' $\mathrm{X}$,' the 'stop' signal, appeared after the go signal. The subjects were told to withhold button press upon seeing the stop signal. Likewise, a trial terminated at button press or when $1 \mathrm{~s}$ had elapsed since the appearance of the stop
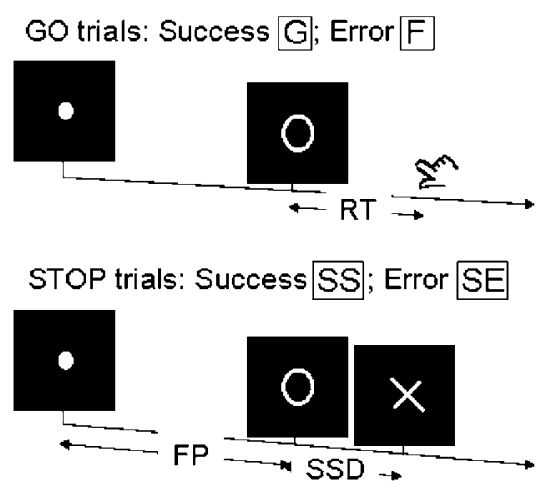

Figure I Stop signal paradigm. In 'go' trials (75\%) observers responded to the go signal (a circle) and in 'stop' trials (25\%) they had to withhold the response when they saw the stop signal (an X). In both trials the go signal appeared after a randomized time interval between I and $5 \mathrm{~s}$ (the foreperiod or FP) following the appearance of the fixation point. The stop signal followed the go signal by a time delay - the stop signal delay (SSD). The SSD was updated according to a staircase procedure, whereby it increased and decreased by $64 \mathrm{~ms}$ following a stop success (SS) and a stop error (SE) trial, respectively. signal. The stop trials constituted the remaining one-quarter of the trials. There was an inter-trial-interval of $2 \mathrm{~s}$.

Clearly, it would be easier for the subject to withhold the response if the stop signal appeared immediately or early after the go signal, and the reverse applied if the time interval between the stop and the go signals (or the stop signal delay, SSD) was extended. The SSD started at $200 \mathrm{~ms}$ and varied from one stop trial to the next according to a staircase procedure: if the subject succeeded in withholding the response, the SSD increased by $64 \mathrm{~ms}$, making it more difficult for them to succeed again in the next stop trial; conversely, if they failed, SSD decreased by $64 \mathrm{~ms}$, making it easier for the next stop trial. With the staircase procedure, a 'critical' SSD could be computed that represents the time delay required for the subject to succeed in withholding a response half of the time in the stop trials (Levitt, 1970). One way to understand the stop signal task is in terms of a horse race model with a go process and a stop process racing toward a finishing line (Logan, 1994). The go process prepares and generates the movement while the stop process inhibits movement initiation: whichever process finishes first determines whether a response will be initiated or not. Importantly, the go and stop processes race toward the activation threshold independently. Thus, the time required for the stop signal to be processed so a response is withheld (ie SSRT) can be computed on the basis of the go trial RT distribution and the odds of successful inhibits for different time delays between go and stop signals. This is done by estimating the critical SSD at which a response can be correctly stopped in approximately $50 \%$ of the stop trials. With the assumptions of this 'horse-race' model, the SSRT could then be computed in the current tracking stop signal task for each individual subject by subtracting the critical SSD from the median go trial RT. Generally speaking, the SSRT is the time required for a subject to cancel the movement after seeing the stop signal. A longer SSRT indicates poor response inhibition.

Subjects were instructed to respond to the go signal quickly while keeping in mind that a stop signal could come up in a small number of trials. Prior to the fMRI study, each subject had a practice session outside the scanner. Each subject completed four 10-min runs of the task with the SSD updated manually across runs. Depending on the actual stimulus timing (eg trials varied in fore-period duration) and speed of response, the total number of trials varied slightly across subjects in an experiment. With the staircase procedure we anticipated that the subjects would succeed in withholding their response in approximately $50 \%$ of the stop trials. This is thus an event-related fMRI study, with the go and stop trials randomly jittered to improve the efficiency of the study design.

After the localization scans but before the blood oxygenation level dependent (BOLD, see below) sessions, subjects were asked to rate their stress level on a Likert scale from 1 (minimal) to 10 (most stressful ever). After they completed the third run of the task, subjects were again asked to rate their frustration and stress level associated with the task on a Likert scale from 1 (minimal) to 10 (most frustrating and stressful ever). The difference in the two ratings was taken to reflect task-related frustration. We also computed a behavioral index of error monitoring. It is known that in a RT task the RT of a correct response is 
prolonged following an error, compared to other correct responses, and this prolonged RT is thought to reflect cognitive processes involved in error monitoring (Rabbit, 1966). We thus computed the RT difference between the go trials that followed a stop error and those that followed another go trial, and termed this RT difference 'post-error slowing' (Hajcak et al, 2003). Both frustration rating and post-error slowing reflected post-response processing.

\section{Imaging Protocol}

Conventional $\mathrm{T}_{1}$-weighted spin-echo sagittal anatomical images were acquired for slice localization using a $3 \mathrm{~T}$ scanner (Siemens Trio). Anatomical images of the functional slice locations were next obtained with spin-echo imaging in the axial plane parallel to the AC-PC line with $\mathrm{TR}=300 \mathrm{~ms}, \mathrm{TE}=2.5 \mathrm{~ms}$, bandwidth $=300 \mathrm{~Hz} /$ pixel, flip angle $=60^{\circ}$, field of view $=220 \times 220 \mathrm{~mm}$, matrix $=$ $256 \times 256,32$ slices with slice thickness $=4 \mathrm{~mm}$ and no gap. Functional, BOLD signals were then acquired with a single-shot gradient-echo echo-planar imaging (EPI) sequence. Thirty-two axial slices parallel to the AC-PC line covering the whole brain were acquired with $\mathrm{TR}=2000 \mathrm{~ms}$, $\mathrm{TE}=25 \mathrm{~ms}$, bandwidth $=2004 \mathrm{~Hz} /$ pixel, flip angle $=85^{\circ}$, field of view $=220 \times 220 \mathrm{~mm}$, matrix $=64 \times 64,32$ slices with slice thickness $=4 \mathrm{~mm}$ and no gap. Three hundred images were acquired in each run for a total of four runs.

\section{Data Analysis and Statistics}

Data were analyzed with Statistical Parametric Mapping version 2 (SPM2, Welcome Department of Imaging Neuroscience, University College London, UK). Images from the first five TRs at the beginning of each trial were discarded to enable the signal to achieve steady-state equilibrium between RF pulsing and relaxation. Images of each individual subject were first corrected for slice timing and realigned (motion-corrected). A mean functional image volume was constructed for each subject for each run from the realigned image volumes. These mean images were normalized to an MNI (Montreal Neurological Institute) EPI template with affine registration followed by nonlinear transformation (Ashburner and Friston, 1999; Friston et al, 1995a). The normalization parameters determined for the mean functional volume were then applied to the corresponding functional image volumes for each subject. Finally, images were smoothed with a Gaussian kernel of $10 \mathrm{~mm}$ at full width at half maximum. The data were highpass filtered $(1 / 128 \mathrm{~Hz}$ cutoff) to remove low-frequency signal drifts.

Four main types of trial outcome were distinguished: go success (G), go error (F), stop success (SS), and stop error (SE) trials (Figure 1). A statistical analytical design was constructed for each individual subject, using the general linear model (GLM) with the onsets of go signal in each of these trial types convolved with a canonical hemodynamic response function (HRF) and with the temporal derivative of the canonical HRF and entered as regressors in the model (Friston et al, 1995b). Additional regressors with the go trial RT and stop trial SSD were also included for parametric modulation. Realignment parameters in all six dimensions were also entered in the model. Serial autocorrelation caused by aliased cardiovascular and respiratory effects was corrected by a first-degree autoregressive or AR(1) model. The GLM estimated the component of variance that could be explained by each of the regressors.

We constructed for each individual subject a single statistical contrast: SS $v s$ SE. Although the stimulus conditions were identical in the two trial types, subjects withheld response in SS but were not able to withhold response in SE trials. Thus, this contrast allowed us to evaluate the relative brain activations during stop signal inhibition. Note that we did not employ a contrast between $G$ and SS (or SE) trials, because cognitive and affective processes other than response inhibition are unequally represented between these trial types; for instance, SS (or $\mathrm{SE}$ ) trials involve more complicated visual processing and emotional frustration than $G$ trials (Li et al, 2006a). In region of interest (ROI) analysis, we used MarsBaR (Brett et al, 2002; http://marsbar.sourceforge.net/) to compute for each individual subject the effect size of activity change for the two ROIs derived from our previous study (Li et al, 2006a). The effect size rather than mean difference in brain activity was derived for correlation with behavioral measures, in order to account for individual differences in the variance of the mean. One ROI focused on the dmFC (Talairach coordinate: $x=-4, y=32, z=51$; Brodmann area or BA 8) and the other focused on the $\operatorname{rACC}(x=-8$, $y=35, z=19$; BA 32/24). By comparing PCD and HC covaried for stop success rate, task-related frustration rating, and post-error slowing, we isolated the neural substrates of impaired impulse control, independent of attentional monitoring (of the stop signal), and post-response processes including affective responses and error monitoring. Specifically, in a covariance analysis, we compared PCD and $\mathrm{HC}$ in brain activation for the contrast SS $>\mathrm{SE}$, with SS rate, frustration rating, and the effect size of post-error slowing as covariates. The covariance analysis was conducted separated for the two ROIs.

\section{RESULTS}

\section{Behavioral Performance}

PCD and HC each successfully completed $97.1 \pm 1.6 \%$ (mean \pm SD) and $97.6 \pm 1.7 \%$ of go trials, $50.9 \pm 1.6 \%$ and $50.1 \pm 1.4 \%$ of stop trials, suggesting the success of the staircase procedure in tracking their performance. PCD and HC did not differ in their median go trial RT $(524 \pm 86 v s$ $565 \pm 122 \mathrm{~ms}, p=0.288$, two-sample $t$ test), post-error slowing in RT (effect size: $2.16 \pm 1.17$ vs $2.40 \pm 1.43, p=0.305$, twosample $t$-test), and task-related frustration rating $(4.3 \pm 2.1 \mathrm{vs}$ $3.3 \pm 1.2$, mean rank $=18$ vs $13, p=0.126$, Mann-Whitney $U$-test). PCD also did not differ from $\mathrm{HC}$ in the SSRT (213 \pm 32 vs $200 \pm 28 \mathrm{~ms}, p=0.236$, two-sample $t$ test).

\section{ROI Analysis}

In ROI analysis, PCD and HC did not differ in the effect size of activation of the dmFC (effect size: $-0.34 \pm 0.84 v s$ $0.63 \pm 2.38, p=0.222$, two-sample $t$-test; Figure $2 \mathrm{a}$ ). However, PCD demonstrated significantly less relative activation than HC in the rACC for the contrast 'SS $>$ SE' (effect size: $-1.38 \pm 2.00$ vs $0.63 \pm 1.56, p<0.005$, two-sample $t$-test; 
a
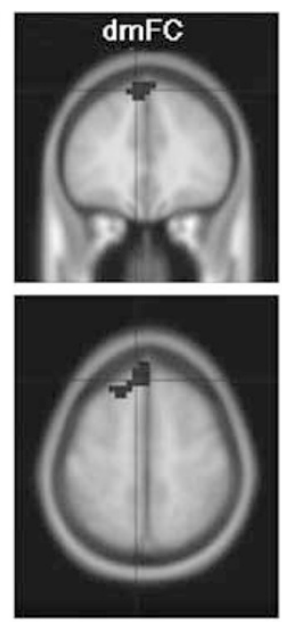

b
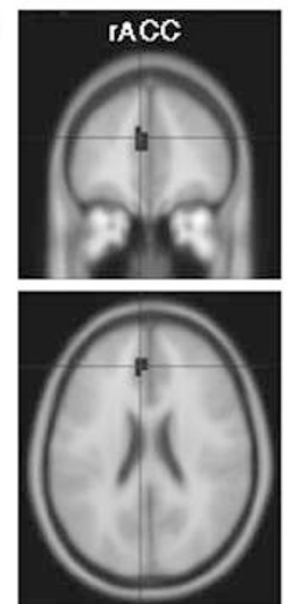
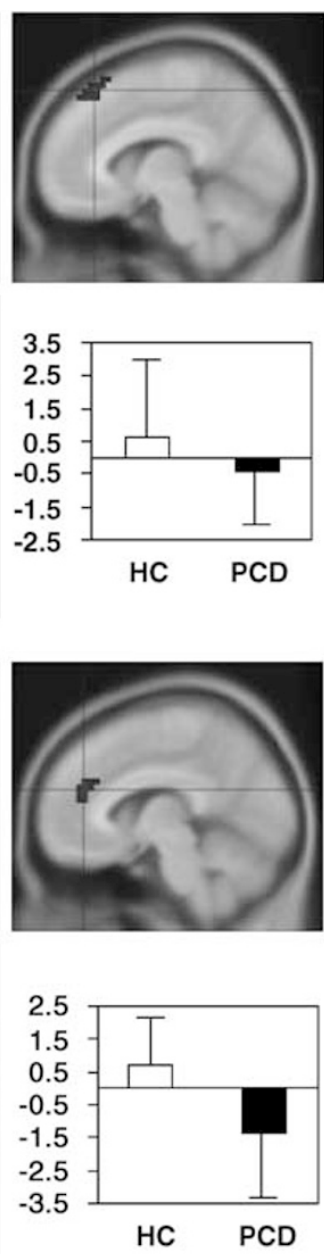
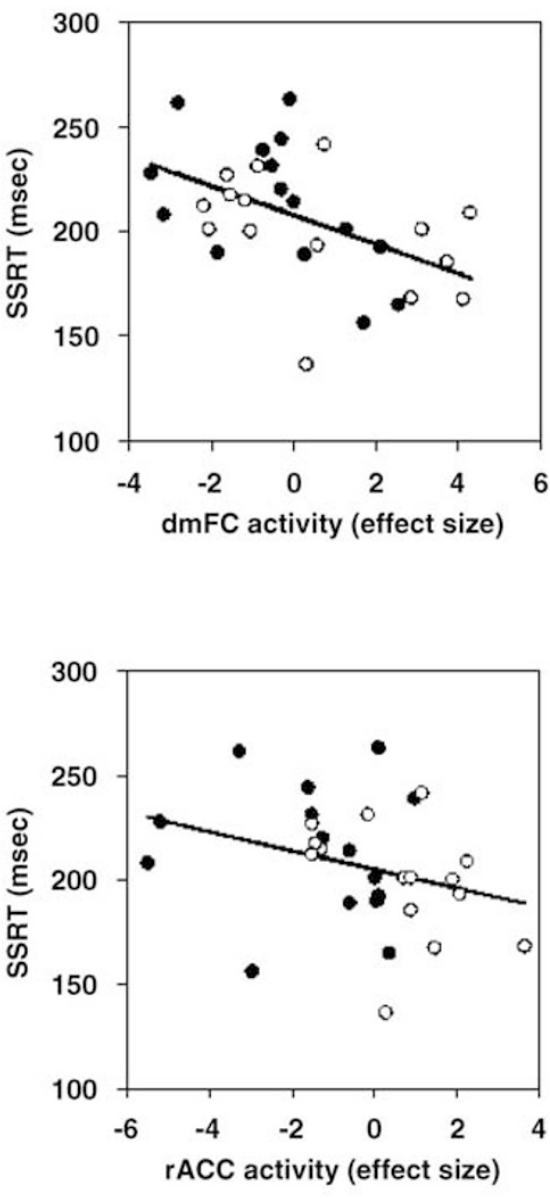

Figure 2 (a) The dorsal medial frontal cortex (dmFC) as an ROI (Talairach coordinate: $x=-4, y=32, z=5 \mathrm{l}$; Li et al, 2006a). The histograms show the effect size (mean \pm SD) for the contrast SS $>$ SE for patients with cocaine dependence (PCD) (filled bar) and healthy control ( $H C$ ) (open bar). PCD and HC did not differ in dmFC activation during stop signal inhibition. On the right, linear regression shows that activation in dmFC correlates inversely with stop signal reaction time (SSRT), consistent with its motor-level role in response inhibition (PCD: filled circles; HC: open circles). (b) The rostral anterior cingulate cortex (rACC) as an ROI (Talairach coordinate: $x=-8, y=35, z=19$; Li et al, 2006a). The histograms show the effect size (mean \pm SD) for the contrast SS $>$ SE for PCD (filled bar) and HC (open bar). PCD showed significantly decreased activation in the rACC, compared to HC, despite nonsignificant difference in SSRT. On the right, linear regression shows that the rACC activation does not correlate with SSRT, consistent with its control-level role in response inhibition (PCD: filled circles; HC: open circles).

Figure 2b). These results remained significant in covariate analysis after controlling for stop success rate, post-error slowing, and task-related frustration rating $(p=0.278$ for $\mathrm{dmFC}$ and $p<0.003$ for rACC). Furthermore, we performed linear regressions of rACC activity (effect size) on these variables to ascertain the independence: $p=0.454$ (stop success rate); $p=0.311$ (frustration rating); $p=0.483$ (posterror slowing). Across subject groups, activation of the dmFC ( $r=-0.497, p=0.005$, Pearson's regression) but not the rACC $(r=0.305, p=0.101)$ correlated with SSRT (Figure 2). When directly compared, the two regressions showed a neartrend level but not significant difference both in slope $(p=0.107)$ and in intercept $(p=0.106$; Zar, 1999).

\section{Neural Correlates of Impulse Control Difficulties in Cocaine Dependence}

To further assess neural correlates of difficulties in impulse control, the relationship between the DERS scores and activity of the two identified ROIs were examined. Total
DERS scores in PCD ( $n=14$, one PCD did not complete the assessment) ranged from 36 to 134 (median $=96.5$ ) and between 6 and 25 (median $=11.5)$ in the impulse control subscale, consistent with our previous report in a larger sample of PCD (average total DERS score $=86$, compared to average total DERS score $=61$ of control subjects, Fox et al, 2007). Linear regression analyses indicated that rACC activity correlated inversely with the DERS total score $(r=-0.541 ; p=0.046$; Figure $3 \mathrm{a})$ and the impulse control subscale score $(r=-0.713 ; p=0.004$; Figure $3 b)$. On the other hand, the dmFC activity did not correlate with either DERS total score $(r=-0.348 ; p=0.223)$ or the impulse control subscale score $(r=-0.501 ; p=0.068)$. A formal comparison, however, showed that the regressions between the two brain regions were not different for either the DERS total score (slope: $p=0.858$; intercept: $p=0.749$ ) or the impulse control subscale score (slope: $p=0.888$; intercept: $p=0.702$; Zar, 1999). We also assessed whether SSRT as a behavioral measure of inhibitory control correlated with these subjective ratings of impulse control difficulties. The 


\section{a}

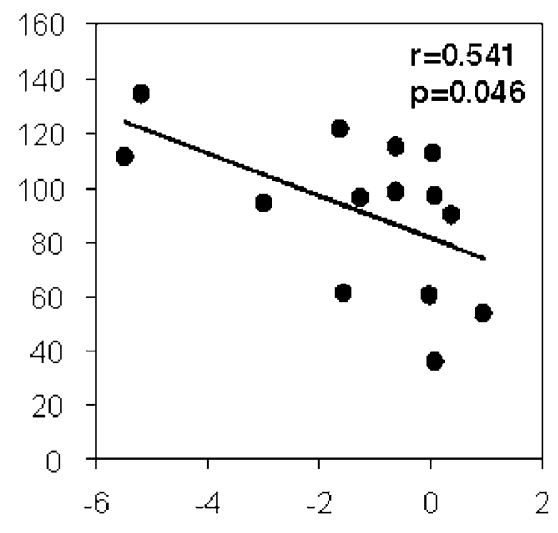

b Impulse control subscore

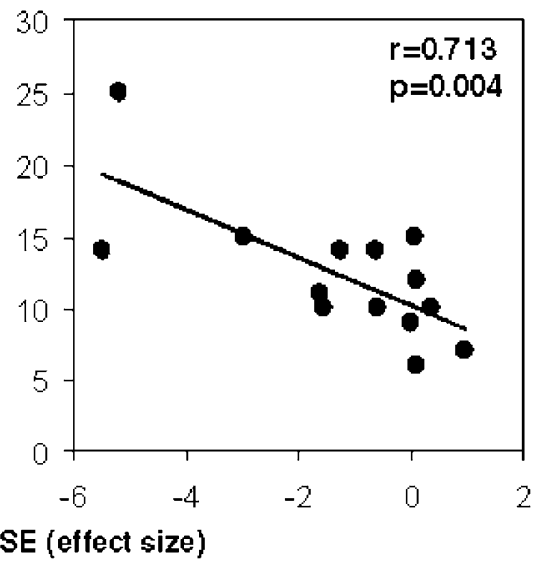

Figure 3 The effect size of activity change in the rostral anterior cingulate cortex (rACC) correlated with total score of the Difficulty in Emotion Regulation Scale (DERS, a) and the impulse control subscale score of Difficulties in Emotion Regulation Scale (DERS) (b).

Table 2 Brain Regions Showing Greater Activation in HC, Compared to PCD

Talairach coordinate $(\mathrm{mm})$

\begin{tabular}{|c|c|c|c|c|c|c|}
\hline $\begin{array}{l}\text { Cluster size } \\
\text { (voxels) }\end{array}$ & Voxel Z-value & $x$ & $y$ & $\mathbf{z}$ & Side & $\begin{array}{l}\text { Brain regions and tentative } \\
\text { Brodmann area (BA) }\end{array}$ \\
\hline \multirow[t]{2}{*}{75} & 4.11 & -50 & -62 & 28 & Left & Angular G, BA 39 \\
\hline & 3.70 & -43 & -46 & 50 & Left & Supramarginal G, BA 40 \\
\hline 15 & 3.62 & -15 & -100 & -9 & Left & Lingual G, BA 17 \\
\hline 14 & 3.39 & -4 & 30 & 20 & Left & Perigenual anterior cingulate G, BA 32/24 \\
\hline \multirow[t]{2}{*}{5} & 3.36 & 41 & -58 & 46 & Right & Supramarginal G, BA 40 \\
\hline & 3.32 & 48 & -50 & 43 & Right & Supramarginal G, BA 40 \\
\hline
\end{tabular}

Abbreviations: $\mathrm{HC}=$ healthy control; $\mathrm{PCD}=$ patients of cocaine dependence.

Note: $p<0.001$, uncorrected and five voxels in extent of activation; all peaked activations $8 \mathrm{~mm}$ apart are identified.

results showed that neither the total score $(r=0.328$; $p=0.252)$ nor the impulse control subscale score $(r=0.243 ; p=0.402)$ correlated with SSRT.

\section{Whole-Brain Comparison between PCD and HC}

No brain regions differed in activity for SS $>$ SE between the two groups at a threshold of $p<0.05$, corrected for familywise error or false discovery rate (Genovese et al, 2002). However, at a lower statistical threshold of $p<0.001$, uncorrected, and five voxels in extent of activation, HC showed greater activation in the visual and posterior parietal regions, compared to PCD, in addition to the rACC (Table 2). Conversely, no brain regions showed greater activation in PCD, as compared to HC.

\section{DISCUSSION}

This study identified relative rACC hypoactivation as a specific deficit in controlling the inhibitory response in men with chronic cocaine abuse. On the basis of the horse race model, we computed the SSRT to index response inhibition in individual subjects. While PCD and HC demonstrated insignificant differences in SSRT, PCD activated the rACC to a lesser extent, compared to $\mathrm{HC}$, during stop signal inhibition. Given the neuroimaging evidence for functional heterogeneity of the ACC and medial frontal cortex, it was important to identify the specific psychological construct underpinning dysfunction in these cortical brain regions (Bush et al, 2000; Ridderinkhof et al, 2004; Rushworth et al, 2004). The cingulate hypoactivation remained significant after controlling for attentional monitoring, post-error behavioral adjustment, and task-related frustration rating, providing ascertaining evidence for a specific role of rACC in mediating inhibitory control. The findings are consistent with earlier fMRI studies and further specify the psychological intermediary of cingulate hypoactivation during the stop signal task (Hester and Garavan, 2004; Kaufman et al, 2003). In particular, the current study focused on the rACC that appears to be in the same locale as reported by Hester and colleagues, an area overlapping both the cognitive and affective subdivisions of the ACC (Bush et al, 2000; Hester and Garavan, 2004). On the other hand, altered activity in 
other subregions of the ACC and other parts of the medial frontal cortex has also been reported in PCD (Adinoff et al, 2003; Bolla et al, 2003; Hester and Garavan, 2004; Kaufman et al, 2003).

The current findings also indicate that the SSRT overlaps significantly between PCD and HC (Figure 2). The SSRT, just as RT in a behavioral task, can be influenced by multiple sensory and motor conditions, and other mental processes (such as effort), that are not directly related to the measure of interest-inhibitory control. Thus, SSRT as a behavioral measure may not capture the between-group differences in response inhibition processes. The current study shows that neuroimaging provides an additional tool to reveal the neural processes associated with impaired response inhibition in PCD. In fact, the observed differences in regional brain activation despite similar behavioral performance are reminiscent of other imaging studies, which purportedly compare groups that are equated in behavioral measures. For instance, a recent study compared prefrontal activities during an n-back memory task between patients with schizophrenia and HCs who were matched in performance under all conditions (Tan et al, 2006). Thus, the observed differences between patients and controls were attributed specifically to the underlying psychopathology of schizophrenia, and the lack of difference in behavioral performance was essential to the validity of such conclusions. Similarly, our current findings provide evidence that the frontal cortical deficits are fundamental to the psychopathology underlying cocaine dependence.

It is worth noting that both in the current and a previous fMRI study, the dmFC activation correlates significantly with SSRT across both controls and PCD, once again supporting the specific role of the dorsal medial frontal region in performing or executing the act of response inhibition (Li et al, 2006a). As described by the horse race model, the SSRT reflects the outcome of an inhibitory control mechanism competing with motor prepotent responses to reach the activation threshold. Connected anatomically with pre-motor and motor structures, the medial frontal region, located in the pre-SMA, is well suited to mediate this motor-level process in response inhibition. In contrast, rACC activation does not correlate with stop signal performance in individual PCD or HC and appears to reflect a process involved in controlling response inhibition, which is not directly related to motor control. On the other hand, the two regressions (dmFC vs SSRT and rACC vs SSRT) were not significantly different in the current work, indicating that the suggested functional differentiation between the two brain regions is merely descriptive and needs to be confirmed in a larger cohort of subjects. Further studies are also warranted to examine whether rACC activity during stop signal inhibition can be generalized beyond the current behavioral task and whether rACC is similarly compromised in PCD during other executive control functions such as those that can be decomposed in the Wisconsin Card Sorting Task (Konishi et al, 2005; Lie et al, 2006).

We showed that rACC activity during stop signal inhibition correlated inversely with the total DERS score and, at greater statistical significance, with the impulse control subscale score. On the other hand, dmFC activity did not demonstrate such associations. These data further support the differentiation of control- and motor-level role of the rACC and the dmFC in mediating stop signal inhibition and more broadly impulse control. The finding also indicates that the rACC activity involving control of an inhibitory response indeed captures aspects of subjective feeling of loss of behavioral control in PCD. However, given that these regressions did not statistically differentiate the two brain regions, further studies would be required to substantiate their functional differences and to explore whether the rACC hypoactivation can be generalized to other cognitive and affective regulation deficits associated with illicit drug use (Meyer-Lindenberg et al, 2006; Sinha et al, 2005; Stadler et al, 2007).

Although we focused on the frontal cortical regions in the current work, whole-brain analyses revealed differences in the posterior parietal and visual cortical activation between PCD and HC. Activation of the posterior parietal lobule (left side, in particular) has been associated with motor attention (see Rushworth et al, 2003 for a review). For instance, both anti-saccade and 'no-go' activated these brain regions to a greater extent then pro-saccade in an oculomotor countermanding task (Brown et al, 2006). Furthermore, we have argued in our previous work that greater visual cortical activation during SS compared to SE trials may reflect greater foveal attention during the stop signal task (Li et al, 2006a). Thus, overall, greater activation of the posterior parietal and visual cortical regions suggest great attention to the task in healthy individuals, whether such attention is directed to change detection or readiness for motor output, compared to cocaine-dependent patients. These results are also consistent with earlier work demonstrating frontal and temporo-parietal hypoperfusion associated with chronic cocaine use (Strickland et al, 1993). Note, however, that a recent study showed greater deactivation of the parietal cortices and activation of the prefrontal and occipital cortices during a visuospatial attention task in cocainedependent patients (Tomasi et al, 2007). Future work with a larger sample size and cross-task comparisons may be required to resolve these potential discrepancies.

It is important to note a few limitations. Firstly, with a small sample size, we were not able to show impaired stop signal performance in the $\mathrm{PCD}$, compared to $\mathrm{HC}$, in the current study. Thus, the present results fall short of ascertaining impaired impulse control in the patients, in contrast to our previous work (Li et al, 2006c). On the other hand, the current imaging findings suggest that the neural correlates of response inhibition provide a more sensitive index of impulse control in cocaine-dependent patients. Secondly, stop signal task is a motor response inhibition task. Despite its mathematical rigor and practicality, the stop signal task does not readily address real-life scenarios of how we exercise inhibitory control. For instance, impulse control is particularly critical for decision making in the context of a reward or stressor. PCD are known to have greater difficulties in delayed gratification. The current results thus represent merely a starting point to investigate these and other factors directly impacting drug using behaviors in the realm of cognitive control. Thirdly, we have not included female subjects in the current study. Women appeared to involve distinct neural circuitry in mediating stop signal inhibition (Li et al, 2006b). Further studies would be warranted to explore whether these 
differences in brain activation during the stop signal task are also seen in women and whether they are related to gender differences in drug using behaviors. Finally, the influence of antisocial personality, which is known to be associated with cocaine use, needs to be addressed in further studies with a larger sample size. Despite these limitations, the current findings are the first to specifically link rACC hypoactivation to controlling the inhibitory response co-varied for attentional monitoring and postresponse processing in abstinent cocaine-dependent men performing a stop signal task. As impulse control difficulties are associated with vulnerability to relapse, identifying its specific neural correlates has potential utility in both the assessment of patients with high impulse control problems and the assessment of new treatments that target improvement in impulse control in men with a history of chronic cocaine abuse.

\section{ACKNOWLEDGEMENTS}

This study was supported by the Yale Interdisciplinary Women's Health Research Scholar Program on Women and Drug Abuse (BIRCWH; K12-DA14038), funded by the NIH Office of Research on Women's Health and the National Institute on Drug Abuse and NIH grants P50-DA16556 (Sinha), K02-DA17232 (Sinha), R03DA022395 (Li), and M01-RR00125 (Yale General Clinical Research Center) to Yale University. This project was also funded in part by the State of Connecticut, Department of Mental Health and Addictions Services.

\section{DISCLOSURE/CONFLICT OF INTEREST}

We declare that over the past 3 years Dr Zubin Bhagwagar has participated on speakers' panels for Bristol-Myers Squibb, Astra Zeneca, and Janssen and has received research support from Bristol-Myers Squibb. We further declare that for Dr Chiang-shan Ray Li, Mr Cong Huang, Ms Peisi Yan, Ms Verica Milivojevic, and Dr Rajita Sinha, except for income received from our primary employer, no financial support or compensation has been received from any individual or corporate entity over the past 3 years for research or professional service and there are no personal financial holdings that could be perceived as constituting a potential conflict of interest.

\section{REFERENCES}

Adinoff B, Devous Sr MD, Cooper DB, Best SE, Chandler P, Harris $\mathrm{T}$ et al (2003). Resting regional cerebral blood flow and gambling task performance in cocaine-dependent subjects and healthy comparison subjects. Am J Psychiatry 160: 1892-1894.

Adinoff B, Williams MJ, Best SE, Harris TS, Chandler P, Devous Sr MD (2006). Sex differences in medial and lateral orbitofrontal cortex hypoperfusion in cocaine-dependent men and women. Gend Med 3: 206-222.

Aron JL, Paulus MP (2007). Location, location: using functional magnetic resonance imaging to pinpoint brain differences relevant to stimulant use. Addiction 102(Suppl 1): 33-43.

Ashburner J, Friston KJ (1999). Nonlinear spatial normalization using basis functions. Hum Brain Mapp 7: 254-266.

Bolla KI, Eldreth DA, London ED, Kiehl KA, Mouratidis M, Contoreggi C et al (2003). Orbitofrontal cortex dysfunction in abstinent cocaine abusers performing a decision-making task. Neuroimage 19: 1085-1094.

Brady KT, Randall CL (1999). Gender differences in substance use disorders. Psychiatr Clin North Am 22: 241-252.

Brett M, Anton J-L, Valabregue R, Poline J-P (2002). Region of interest analysis using an SPM toolbox. Abstract presented at the 8th International Conference on Functional Mapping of the Human Brain, June 2-6, Sendai, Japan.

Brown MR, Goltz HC, Vilis T, Ford KA, Everling S (2006). Inhibition and generation of saccades: rapid event-related fMRI of prosaccades, antisaccades, and nogo trials. Neuroimage 33: 644-659.

Bush G, Luu P, Posner MI (2000). Cognitive and emotional influences in anterior cingulate cortex. Trend Cognit Sci 4: 215-222.

Dawe S, Loxton NJ (2004). The role of impulsivity in the development of substance use and eating disorders. Neurosci Biobehav Rev 28: 343-351.

Dougherty DM, Mathias CW, Marsh DM, Moeller FG, Swann AC (2004). Suicidal behaviors and drug abuse: impulsivity and its assessment. Drug Alcohol Depend 76: S93-S105.

Ernst M, Paulus MP (2005). Neurobiology of decision making: a selective review from a neurocognitive and clinical perspective. Biol Psychiatry 58: 597-604.

Everitt BJ, Robbins TW (2005). Neural systems of reinforcement for drug addiction: from actions to habits to compulsion. Nat Rev Neurosci 8: 1481-1489.

First MB, Spitzer RL, Williams JBW, Gibbon M (1995). Structured Clinical Interview for DSM-IV (SCID). American Psychiatric Association: Washington DC.

Fox HC, Axelrod SR, Paliwal P, Sleeper J, Sinha R (2007). Difficulties in emotion regulation and impulse control during cocaine abstinence. Drug Alcoh Depend 89: 298-301.

Friston KJ, Ashburner J, Frith CD, Polone J-B, Heather JD, Frackowiak RSJ (1995a). Spatial registration and normalization of images. Hum Brain Mapp 2: 165-189.

Friston KJ, Holmes AP, Worsley KJ, Poline J-B, Frith CD, Frackowiak RSJ (1995b). Statistical parametric maps in functional imaging: a general linear approach. Hum Brain Mapp 2: $189-210$.

Genovese CR, Lazar NA, Nichols T (2002). Thresholding of statistical maps in functional neuroimaging using the false discovery rate. Neuroimage 15: 870-878.

Goldstein JM, Jerram M, Poldrack R, Anagnoson R, Breiter HC, Makris $\mathrm{N}$ et al (2005). Sex differences in prefrontal cortical brain activity during fMRI of auditory verbal working memory. Neuropsychology 19: 509-519.

Goldstein RZ, Volkow ND (2002). Drug addiction and its underlying neurobiological basis: neuroimaging evidence for the involvement of the frontal cortex. Am J Psychiatry 159: $1642-1652$.

Gratz KL, Roemer L (2004). Multidimensional assessment of emotion regulation and dsyregulation: development, factor structure, and initial validation of the difficulties in emotion regulation scale. J Psychopathol Behav Assess 26: 41-54.

Hajcak G, McDonald N, Simons RF (2003). To err is autonomic: error-related brain potentials, ANS activity, and post-error compensatory behavior. Psychophysiol 40: 895-903.

Hayaki J, Stein MD, Lassor JA, Herman DS, Anderson BJ (2005). Adversity among drug users: relationship to impulsivity. Drug Alcohol Depend 78: 65-71.

Hester R, Garavan H (2004). Executive dysfunction in cocaine addiction: evidence for discordant frontal, cingulate, and cerebellar activity. J Neurosci 24: 11017-11022.

Jovanovski D, Erb S, Zakzanis KK (2005). Neurocognitive deficits in cocaine users: a quantitative review of the evidence. J Clin Exp Neuropsychol 27: 189-204.

Kaufman JN, Ross TJ, Stein EA, Garavan H (2003). Cingulate hypoactivity in cocaine users during a GO-NOGO task as 
revealed by event-related functional magnetic resonance imaging. J Neurosci 23: 7839-7843.

Kim SJ, Lyoo IK, Hwang J, Sung YH, Lee HY, Lee DS et al (2005). Frontal glucose hypometabolism in abstinent methamphetamine users. Neuropsychopharmacol 30: 1383-1391.

Konishi S, Chikazoe J, Jimura K, Asari T, Miyashita Y (2005). Neural mechanism in anterior prefrontal cortex for inhibition of prolonged set interference. Proc Natl Acad Sci USA 102: 12584-12588.

Levitt H (1970). Transformed up-down methods in psychoacoustics. J Acoust Soc Am 49: 467-477.

Li C-SR, Huang C, Constable RT, Sinha R (2006a). Imaging response inhibition in a stop signal task-neural correlates independent of signal monitoring and post-response processing. J Neurosci 26: 186-192.

Li C-SR, Huang C, Constable RT, Sinha R (2006b). Gender differences in the neural correlates of response inhibition in a stop signal task. Neuroimage 32: 1918-1929.

Li C-SR, Kemp K, Milivojevic V, Sinha R (2005a). Neuroimaging study of sex differences in the neuropathology of cocaine abuse. Gend Med 2: 174-182.

Li C-SR, Kosten TR, Sinha R (2005b). Sex differences in brain activation during stress imagery in abstinent cocaine users: a functional magnetic resonance imaging study. Biol Psychiatry 57: 487-494.

Li C-SR, Krystal JH, Mathalon DH (2005c). Fore-period effect and stop signal processing time. Exp Brain Res 167: 305-309.

Li C-SR, Milivojevic V, Kemp KA, Hong K, Sinha R (2006c). Performance monitoring and stop signal inhibition in abstinent patients with cocaine dependence. Drug Alcoh Depend 85: 205-212.

Lie CH, Specht K, Marshall JC, Fink GR (2006). Using fMRI to decompose the neural processes underlying the Wisconsin Card Sorting Test. Neuroimage 30: 1038-1049.

Logan GD (1994). Inhibitory processes in attention, memory and language. In: Dagenbach $\mathrm{D}$, Carr $\mathrm{TH}$ (eds). On the Ability to Inhibit Thought and Action: A User's Guide to the Stop Signal Paradigm. Academic Press: San Diego. pp 189-239.

Meyer-Lindenberg A, Buckholtz JW, Kolachana B, R Hariri A, Pezawas L, Blasi G et al (2006). Neural mechanisms of genetic risk for impulsivity and violence in humans. Proc Natl Acad Sci USA 103: 6269-6274.

Moeller FG, Barratt ES, Dougherty DM, Schmitz JM, Swann AC (2001). Psychiatric aspects of impulsivity. Am J Psychiatry 158: 1783-1793.
Rabbit PMA (1966). Errors and error correction in choice-response tasks. J Exp Psychol 71: 264-272.

Ridderinkhof KR, Ullsperger M, Crone EA, Nieuwenhuis S (2004). The role of the medial frontal cortex in cognitive control. Science 306: 443-447.

Rushworth MF, Johansen-Berg H, Göbel SM, Devlin JT (2003). The left parietal and premotor cortices: motor attention and selection. Neuroimage 20(Suppl 1): S89-100.

Rushworth MFS, Walton ME, Kennerley SW, Bannerman DM (2004). Action sets and decisions in the medial frontal cortex. Trend Cognit Sci 8: 410-417.

Sinha R, Lacadie C, Skudlarski P, Fulbright RK, Rounsaville BJ, Kosten TR et al (2005). Neural activity associated with stressinduced cocaine craving: a functional magnetic resonance imaging study. Psychopharmacol (Berl) 183: 171-180.

Sinha R, Rounsaville BJ (2002). Sex differences in depressed substance abusers. J Clin Psychiatry 63: 616-627.

Stadler C, Sterzer P, Schmeck K, Krebs A, Kleinschmidt A, Poustka F (2007). Reduced anterior cingulate activation in aggressive children and adolescents during affective stimulation: association with temperament traits. J Psychiatr Res 41: 410-417.

Strickland TL, Mena I, Villanueva-Meyer J, Miller BL, Cummings J, Mehringer CM et al (1993). Cerebral perfusion and neuropsychological consequences of chronic cocaine use. J Neuropsychiatry Clin Neurosci 5: 419-427.

Tan HY, Sust S, Buckholtz JW, Mattay VS, Meyer-Lindenberg A, Egan MF et al (2006). Dysfunctional prefrontal regional specialization and compensation in schizophrenia. Am J Psychiatry 163: 1969-1977.

Tomasi D, Goldstein RZ, Telang F, Maloney T, Alia-Klein N, Caparelli EC et al (2007). Thalamo-cortical dysfunction in cocaine abusers: implications in attention and perception. Psychiatry Res 155: 189-201.

Verdejo-García A, Bechara A, Recknor EC, Pérez-García M (2006). Executive dysfunction in substance dependent individuals during drug use and abstinence: an examination of the behavioral, cognitive and emotional correlates of addiction. J Int Neuropsychol Soc 12: 405-415.

Wager TD, Phan KL, Liberzon I, Taylor SF (2003). Valence, gender, and lateralization of functional brain anatomy in emotion: a meta-analysis of findings from neuroimaging. Neuroimage 19: 513-531.

Zar JH (1999). Biostatistical Analysis. Prentice Hall, Upper Saddle River: New Jersey. pp 360-376. 